\title{
Effective Suppression of Hypoxia Induced Angiogenesis by Synergism of Simvastatin and Metformin via Inhibiting ET-1-ETBR-Hif1a Signaling Axis
}

Jie Liu

Xi'an Jiaotong University

Huxia Wang

Xi'an Jiaotong University

Miao Zhang

Xi'an Jiaotong University

Yazhao Li

Xi'an Jiaotong University

Ruiqi Wang

Xi'an Jiaotong University

He Chen

Xi'an Jiaotong University

Bo Wang

Xi'an Jiaotong University

Xiaoqian Gao

Xi'an Jiaotong University

Shaoran Song

Xi'an Jiaotong University

Jichang Wang

Xi'an Jiaotong University

Yaochun Wang

Xi'an Jiaotong University

Juan Li

Xi'an Jiaotong University

Peijun Liu ( $\square$ liupeijun@mail.xjtu.edu.cn )

Xi'an Jiaotong University https://orcid.org/0000-0002-0767-0774

Research 
Keywords: Hypoxia, simvastatin, metformin, ET-1, ETBR, Hif1a

Posted Date: September 20th, 2021

DOI: https://doi.org/10.21203/rs.3.rs-885738/v1

License: (c) (1) This work is licensed under a Creative Commons Attribution 4.0 International License. Read Full License 


\section{Abstract}

Background: Hypoxic stress accelerates metabolic reprogramming to promote malignant progression, which contains huge and interesting drug targets for cancer therapy. According to recently reports and our previously researches, simvastatin (SVA) or metformin (MET), two metabolic relevant drugs, play an important role in inhibiting tumor growth, angiogenesis, improvement hypoxic microenvironment. However, the signal networks of hypoxia induced metabolic reprogramming is enormous and intricacy, blocking a signal target can't play an effective role in antitumor, whether combination of SVA and MET could exert a more effective antitumor effect is not clear.

Methods: The antitumor effect of synergism of SVA and MET were detected in mouse models, breast cancer patient-derived organoid (PDO) and multiple tumor cell lines compared with untreated, SVA or MET alone. Transcriptome sequencing were performed for exploring the possible mechanisms. Inhibition of endothelin 1 (ET-1) induced Hif1a by synergism of SVA and MET were contrasted with bosentan, an ETBR antagonist.

Results: Our results show that synergism of SVA and MET can inhibit tumor cell proliferation and promote apoptosis, alleviate hypoxia, decrease angiogenesis and increase vessel normalization within tumor than use of SVA or MET alone. RNA-sequencing result implies that just synergism of SVA and MET can inhibit EDN1 expression, which encodes endothelin 1, an important regulator of angiogenesis and hypoxia-related pathway, but not in use of SVA or MET alone. More specifically, synergism of SVA and MET suppresses the ET-1 induced Hif1a expression by inhibiting ET-1-ETBR signaling pathway and increasing PHD2 expression. In addition, the antitumor effect of synergism of SVA and MET is more efficient than bosentan in tumor cells and breast cancer PDO by inhibiting ET-1-ETBR-Hif1a signaling axis.

Conclusions: Thus, our data show that synergism of SVA and MET can be a viable therapy for antitumor.

\section{Background}

Metabolic reprogramming is a hallmark of malignancy and an important breakthrough point of tumor therapy $[1,2]$. In order to satisfy their own rapid proliferation, tumor cells reprogrammed the stereotyped metabolic activities, notably as aerobic glycolysis, glutamine catabolism, lipid metabolism, macromolecular synthesis, and redox homeostasis $[3,4]$. They rearrange and deploy the signaling pathway and network of these metabolic activities $[5,6]$. Hypoxia response system facilitates metabolic reprogramming in many tumors, the hypoxic cancer cells depend on glucose to cause lactate accumulation. Hypoxia-inducible factor $1 \mathrm{a}(\mathrm{HIF} 1 \mathrm{a})$ is one of the most important primary transcriptional response factor of hypoxic stress and broadly increased expression in many human cancers, and closely correlate with tumor initiation, growth, metabolism, angiogenesis, invasion, inflammatory cell recruitment and metastasis and poor patient prognosis [7-9]. Thus, a variety of tumor therapies and inhibitors have 
been designed to target these metabolic pathways, and many of which have started clinical trials $[5,10$, 11], but it still needs long-time and more in-depth research and efforts.

Statins, such as simvastatin (SVA), the first-line clinical medication for decreasing cholesterol levels and cardiovascular disease. Many researches and our previously studies showed that statins also revealed antitumor effects, such as preventing colorectal cancer, inhibiting the proliferation of breast cancer cells and invasion and metastasis of human melanoma cells [12-16]. In addition, metformin (MET) can inhibit growth of many types' tumors, such as breast cancer, hepatocellular carcinoma. Metformin is the first-line clinical medication for type 2 diabetes, nowadays its play a role in antitumor via inhibiting Warburg effect and alleviating hypoxia [17-20]. Whether both inhibiting the glucose and lipid metabolism of tumor cells by combination of SVA and MET have more effective than SVA and MET alone use for antitumor effect?

In this study, we found SVA and MET exert a synergistic effect to significantly inhibit the growth of tumors and breast cancer patient-derived organoid (PDO), and induce the apoptosis of tumor cells, as well as alleviate hypoxia, inhibit angiogenesis and increase vessel normalization within the tumors than SVA or MET alone. Mainly, the synergistic effect inhibits the activation of ET-1-Hif1a axis. The synergism of SVA and MET will became a potential therapeutic strategy for anti-tumor treatment.

\section{Methods}

\section{Cell culture and hypoxia mode}

HepG2, Hep3B, MCF7, MDA-MB-231, H1299, SiHa, C33A and 4T1 cells were cultured in high glucose Dulbecco 's Modified Eagle 's medium (HyClone, Logan UT, USA) and 10\% fetal bovine serum (HyClone, Logan UT, USA). A549 and B16 cells were cultured in RPMI-1640 medium (HyClone, Logan UT, USA) and $10 \%$ fetal bovine serum. All cells were maintained at $37{ }^{\circ} \mathrm{C}$ and $5 \% \mathrm{CO}_{2}$ in incubator. All cell lines were obtained from China Infrastructure of Cell Line Resource (Beijing, China)

Cells were cultured in a $1 \% \mathrm{O}_{2}, 5 \% \mathrm{CO}_{2}$ incubator for $24 \mathrm{~h}$ to establish a physical anoxia state.

\section{Colony formation}

Cells were treated with SVA and/or MET for 24 hours and then reseeded in new dishes for 14 days. The cells were fixed using $4 \%$ paraformaldehyde (PFA) and then stained with $0.5 \%$ crystal violet.

\section{Carboxyfluorescein diacetate succinimidyl ester (CFSE) assay}

Cells were stained with $2.5 \mu \mathrm{M}$ CFSE for $30 \mathrm{~min}$ and then treated with relevant concentration SVA and/or MET, analyzed by flow cytometry (BD Biosciences, USA). The protocol was described as previously [19].

\section{Apoptosis assays}


After treatment with SVA and/or MET, cells were collected and followed the Annexin V-PE apoptosis detection kit (BD Biosciences, USA) to analysis the percentage of apoptosis cells.

\section{RNA sequencing assay}

HepG2 cells were untreated or treated with $2.5 \mu \mathrm{M}$ SVA, 5mM MET and combination of $2.5 \mu \mathrm{M}$ SVA and $5 \mathrm{mM}$ MET for $24 \mathrm{~h}$, quickly extracted and cryopreserved with liquid nitrogen. Then RNA sequencing were performed by Guangzhou RiboBio (China, Guangzhou). Every group were contain 3 independent samples for gene expression analysis.

\section{Mouse models}

All animal procedures were performed according to the protocol approved by the Institutional Animal Care and Use Committee at Xi 'an Jiaotong University. The $5 \times 10^{5} 4 \mathrm{~T} 1$ cells were injected into the fat pad of the $4^{\text {th }}$ left mammary of four-week-old female BALB/c mice, and $1 \times 10^{6}$ B16 cells were subcutaneously injected into four-week-old female C57BL/6J mice. And the mice were treated with $15 \mathrm{mg} / \mathrm{kg} / \mathrm{day}$ simvastatin and/or $100 \mathrm{mg} / \mathrm{kg} /$ day metformin by intragastric administration.

\section{Immunoblotting}

After treatment with SVA and/or MET, cells were harvested by RIPA buffer, and the lysates were underwent SDS-PAGE and transferred onto PVDF membrane. Then the membrane were incubated with primary antibody and HRP-conjugated sencondary antibody, and the chemiluminescent signals were detected by ChemiDoc $^{\text {TM }}$ XRS+ (Bio-rad, USA). The antibodies anti-p-RB, anti-RB, anti-cleaved caspase3, anticaspase3, anti-Hif1a were obtained from Cell Signaling Technology (USA), anti-MCM7, anti-ET-1, antiPHD2 were purchased from Santa Cruz (USA), anti-ETAR and anti-ETBR were purchased from Abcam (UK), and surviving were purchased from Proteintech (China).

\section{Immunofluorescence and immunohistochemistry}

Cells or mouse tissues were fixed by $4 \%$ paraformaldehyde (PFA) and permeabilized with $0.5 \%$ Triton X100 in PBS, followed blocking with $5 \%$ BSA, supplemented with $10 \%$ goat serum. Subsequently the slides were stained with CD31 (Abcam, UK), NG2 (BD Biosciences, USA), VE-cadherin (Biolegend, USA), Hif1a (Cell Signaling Technology, USA) and ETBR (Abcam, UK). Nuclei were stained with $5 \mu \mathrm{g} / \mathrm{mL} 4,6$ diamidino-2-phenylindone (DAPI). The images were taken by Leica TCS SP5 confocal laser scanning microscopy (Leica, Germany). The detailed procedures were similarly performed as described previously [19].

The perfusion status of vessels were labeled by Rhodamine-labeled lectin (Vector Labs, USA) for 15 min. $20 \mathrm{mg} / \mathrm{kg}$ Lectin were intravenous injected into mice, then intracardiac perfusion of $40 \mathrm{ml} \mathrm{4 \%}$ PFA with a flux of $10 \mathrm{ml} / \mathrm{min}$. Tumors were extracted and fixed with 4\% PFA, embedded in OCT (Sakura Finetek, USA), and produced frozen sections. Finally, co-staining with CD31 and imaging with confocal laser scanning microscopy. 
Tissues were fixed in $10 \%$ neutralized formaldehyde and embedded in paraffin, and then stained with PCNA (Santa Cruz, USA), cleaved caspase3 and Hif1a. Images were taken by Leica SCN400 slide scanner (Leica, Germany). The detailed procedures were similarly performed as described previously $[19,20]$.

\section{Patient-derived organoid model}

Breast cancer tissues were obtained from First Affiliated Hospital of Xi 'an Jiaotong University with informed consent and approved by the Ethics Review Committee of the First Affiliated Hospital of Xi 'an Jiaotong University. The breast cancer tissues were chopped up and digested by Collagenase at $37{ }^{\circ} \mathrm{C}$ for $1 \mathrm{~h}$ followed by filtering on a 150 mesh filter and centrifuging $10 \mathrm{~min}$ at $400 \mathrm{~g}$. The precipitate was

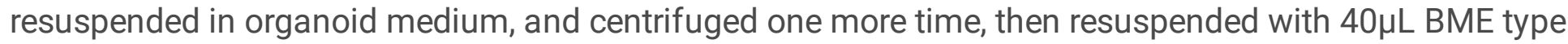
2 (Trevigen, USA). After BME was solidified, $400 \mu \mathrm{L}$ organoid medium was added for culture. The detailed procedures and reagents were performed as described previously [21]

The viability were assayed as followed: the PDOs were dispensed into 384-well microplates and treated with SVA and/or MET for $0,24 \mathrm{~h}, 48$ or 72h, then analyzed by CellTiter-Glo (Promega, USA) accordance with its' technical manual.

\section{Statistical analysis}

The quantitative analysis performed by Prism 7.0 software, and the quantitative data were represented by mean \pm SEM. Statistical significance was calculated using two-tailed student ' $s \mathrm{t}$ test or one-way ANOVA t-test. Each experiment data was represented a minimum of three biological replicates. ${ }^{\star} P<0.05, * \star P<$ $0.01, * \star * P<0.001$.

\section{Results}

\section{Combination treatment of simvastatin and metformin inhibits tumor growth in vivo.}

Malignancy could reprogram their metabolism and utilize glucose and lipid for their high energy demand [3]. According to our previous research and recently reports, treated by MET [17-20] or SVA [12-14, 16] alone could inhibit growth and induce apoptosis of tumor cells both in vivo and in vitro [22]. These two antitumor drugs are mainly clinically used to reduce the glucose and lipid [16, 23-25]. Based on this, we found there was an observably inhibition of tumor growth and prolong the survival duration of mice after the treatment with combination of SVA and MET. Not only compared with untreated group, but also used SVA or MET alone, the combination treatment displayed a significant antitumor effect (Fig. 1A-F). The combination treatment decreased the expression of proliferating cell nuclear antigen (PCNA) and increased cleaved caspase 3 expression of the 4T1 and B16 tumors than other group (Fig. 1G, H, sFig1), it suggested that combination treatment could inhibit the proliferation and induce apoptosis of tumor cells.

\section{Combination treatment of simvastatin and metformin is a synergism for antitumor effect}


Similar to the in vivo, combination treatment of simvastatin and metformin also inhibited proliferation of poly-type of tumor cells in vitro (Fig. 2A, B, sFig 2, Table 1), which displayed a moderate to strong synergism [26]. According to our preciously study, SVA can inhibit phosphorylated retinoblastoma (RB) and mini-chromosome maintenance protein 7 (MCM7) expression to suppression of tumor cell growth [13]. The synergism of simvastatin and metformin inhibited the tumor cell proliferation via decreasing the p-RB and MCM7 (Fig. 2C). It could induce 2-3 times apoptosis ratio than other treatment, accompanied by upregulating of cleaved caspase 3 and downregulating survivin (Fig. 2D-F). Thus, we found SVA and MET synergistically suppressed cell proliferation and induced apoptosis.

Table 1

Drug combinations

\begin{tabular}{|lll|}
\hline Cell lines & Combination index $\left(\mathrm{Cl}_{50}\right)^{\mathrm{a}}$ & \\
\hline HepG2 & 0.458 & synergism \\
\hline Hep3B & 0.325 & strong synergism \\
\hline MCF7 & 0.5 & synergism \\
\hline MDA-MB-231 & 0.625 & moderate synergism \\
A549 & 0.35 & strong synergism \\
$\mathrm{H} 1299$ & 0.375 & strong synergism \\
SiHa & 0.72 & moderate synergism \\
\hline $\mathrm{C} 33 \mathrm{~A}$ & 0.75 & moderate synergism \\
\hline $\begin{array}{l}\text { a } \mathrm{Cl}_{50}>1.3 \text { (antagonism) } ; 1.3 \geq \mathrm{Cl}_{50}>1.1 \text { (moderate antagonism) } ; 1.1 \geq \mathrm{Cl}_{50}>0.9 \text { (additive effect); } \\
0.9 \geq \mathrm{Cl}_{50}>0.8 \text { (slight synergism); } 0.8 \geq \mathrm{Cl}_{50}>0.6 \text { (moderate synergism); } 0.6 \geq \mathrm{Cl}_{50}>0.4 \text { (synergism); } \\
0.4 \geq \mathrm{Cl}_{50}>0.2 \text { (strong synergism). }\end{array}$ \\
\hline
\end{tabular}

\section{Synergism of simvastatin and metformin inhibits angiogenesis and promotes vcessel normalization}

To investigate the mechanism of synergism of SVA and MET in antitumor effect, we applied RNA sequencing to computationally investigate transcriptional dynamics after treatment of synergism of SVA and MET, SVA or MET alone and their control in HepG2 cells. Then we predicted the altered biological process of synergism alone, but no distinction in treatment after SVA or MET alone and their control. Compared with negative control, SVA or MET, synergism of SVA and MET changed lots of angiogenesis related genes (Fig. 3A). Angiogenesis is required for caner progression, thus antiangiogenic therapy a widely used in antitumor therapy [27-29]. Platelet endothelial cell adhesion molecule (PECAM-1) (CD31) can marker the endothelial cell of vessel [30]. Synergetic treatment decreased the number and branch points of tumor inside vessel than others by CD31-staining (Fig. 3B-G). It suggested that synergism of SVA or MET could inhibits tumor angiogenesis. 
Inhibition of tumor angiogenesis is the key point of the hypothesis about tumor-starving, vascular endothelial growth factor (VEGF) as a major driver of it, became a notable target for antitumor. However, in preclinical studies anti-VEGF therapy can induce tumor vasculature showing a more "mature" or "normal" phenotype. The vessel normalization display an attenuated hyperpermeability, increased vascular pericyte coverage, and a more normal basement membrane [30]. Here, we found the length of CD31-stained vessels after synergism was longer than other treatments (Fig. 3B, E). NG2 is the marker of pericytes, and vascular endothelial (VE)-cadherin is the strictly endothelial specific adhesion molecule [30], both of them were increased and colocalization with CD31 after synergetic treatment of SVA and MET (Fig. 4A-D). Lectin can bind to the surface of endothelial cells accompanied with blood flow. Lectin ${ }^{+}$ vessels also increased after synergism of SVA and MET (Fig. 4E, F). Synergism of SVA and MET inhibited disorganized and nonfunctional vessels and promoted vessel normalization and maturity.

\section{Simvastatin and metformin synergistically promotes vessel normalization via inhibiting ET-1-Hif1a signaling axis}

KEGG pathway analysis showed that activity of relaxin and Hif1a signaling pathway were changed after synergism of SVA and MET. EDN1 markedly decreased after synergism treatment, which encodes endothelin 1 (ET-1) [31], and play an important role in regulating Hif1a signaling pathway (Fig. 5A). ET-1 and its receptor endothelin receptor $B$ (ETBR) were reduced by synergism treatment, but it had no effect on receptor endothelin receptor A (ETAR) (Fig. 5B-D). Subsequently, we analyzed the hypoxic area by staining of Hif1a in 4T1 tumor and B16 tumor. Synergism treatment decreased more than 50\% hypoxic area than untreated group, and $25 \%-35 \%$ than simvastatin or metformin treatment groups in $4 \mathrm{t} 1$ tumors; decreased more than $60 \%$ hypoxic area than untreated group, and $40 \%-50 \%$ than simvastatin or metformin treatment groups in B16 tumors (Fig. 5E, F). Similar to in vivo, synergism treatment inhibited Hif1 a expression during hypoxic condition $\left(1 \% \mathrm{O}_{2}\right)$ (Fig. $5 \mathrm{G}$ ).

Under normoxic conditions, prolyl hydroxylase 2 (PHD2) catalyzes hydroxylation of HIF1a to lead its proteasomal degradation [8]. Endothelin 1 induced Hif1 $a$ and inhibited PHD2 expression associated with up-regulated expression of ETBR in HepG2 and MCF7 cells (Fig. 6A). Synergism treatment shown a significant inhibition of ET-1 induced ETBR and Hif1a expression, accompanied with promoting PDH2 expression (Fig. 6B). Furthermore, ET-1 induced Hif1a expression shown a nuclear location, which also could be inhibited by synergism treatment (Fig. 6C, sFig 3). Bosentan is an endothelin receptor antagonist, targeted ETAR and ETBR, and have been evaluated in clinical oncology [32]. Synergism treatment could suppress ET-1 induced Hif1a and ETBR expression similar to inhibition of ETBR expression by bosentan or small interfering RNAs (siRNAs) (Fig. 6D, sFig 4). Compared with treatment by bosentan, synergism of SVA and MET displayed a stronger inhibition cell proliferation by decreasing ET-1 induced Hif1a expression and correspondent PHD2 expression (Fig. 6D-E). It suggested that inhibiting ET1 induced Hif1a expression could become one important pathway to suppress angiogenesis and promoted vessel normalization by synergism of simvastatin and metformin. 


\section{Synergism of simvastatin and metformin suppresses growth and ET-1 induced Hif1a expression of breast cancer PDOs}

PDO models link cancer genetics and patient trial, cover the shortage of cell-line- and xenograft-based drug research, directly and rapidly test drug sensitivity, and promote personalized tumor therapy [21, 33]. From 8 tumors, we established breast cancer PDOs that readily expanded. Based on the results of hematoxylin-eosin (H\&E) staining of paraffin section, the histopathological features of breast cancer PDOs shown a strong concordance with of their corresponding parental tumor (Fig. 7A, sTab 1). There were dramatically inhibited the viability and volume of breast cancer PDOs after three days of synergism of SVA and MET treatment than vehicle, simvastatin or metformin alone (Fig. 7B-D). We also found the expression of ET-1 and ETBR were suppressed by synergism treatment (Fig. 7E, F). Similarly to the in vitro, the ET-1 induced Hif1a expression were inhibited by bosentan or synergism (Fig. 7G). Thus, we found that inhibition of ET-1 induced Hif1a expression is a significant mechanism of synergism of SVA and MET to anti-tumor effect (Fig. 7H).

\section{Discussion}

Our studies demonstrate that simvastatin and metformin shown an effective synergism in anti-tumor, which can inhibit growth of tumor cells, angiogenesis and promote vessel normalization by inhibiting ET1 expression and ET-1 induced Hif1a expression, than SVA or MET alone. Several researches were focused on the efficacy of simvastatin plus metformin treatment in polycystic ovary syndrome [34]. Others were mainly about anti-tumor effect, such as inhibition of prostate cancer, colorectal cancer, hepatocellular carcinoma and endometrial cancer, etc [35-42]. In our study, we also found that combination of simvastatin and metformin had a wide range of anti-cancer effects in breast cancer, hepatocellular carcinoma, lung cancer and cervical cancer. And the widespread anti-tumor effect of SVA plus MET is not a simple superposition, but a moderate to strong synergism than use of SVA or MET alone.

Study on combination SVA and MET in anti-tumor effect, mainly focused on inducing apoptosis and inhibition proliferation. Similarly to our results, in endometrial cancer [36], combination SVA and MET synergistically induced apoptosis and inhibited cell growth, which could be reversed by silencing Bim expression [36]. In metastatic castration-resistant prostate cancer cells, it inhibits cell viability and metastasis and increases Ripk1- and Ripk3-dependent necrotic cell death after 48-96h treatment by combination of simvastatin and metformin, but not induces apoptosis [35]. The duration of treatment and tissue differential expression may be mainly reason of the different effect of simvastatin and metformin. In hepatoma cells, combination of metformin and simvastatin decreased cell proliferation and arresting cell cycle in G0/G1 by inhibiting translational controlled tumor protein (TCTP) and mammalian target of rapamycin (mTOR) pathway and increasing tumor suppressor phosphatase and tensin homolog (PTEN) and promoting autophagy [42]. Combination of SVA and MET inhibited primary ventral prostate tumor formation, cachexia, bone metastasis, and biochemical failure by ameliorating metabolic aberrations and increasing AMPKa catalytic activity, more effectively than SVA, MET treatment 
alone or docetaxel (DTX) chemotherapy, the standard-of-care of metastatic castration-resistant prostate cancer (CRPC). They also found SVA and MET combination may have a broader applicability in triplenegative breast cancer [35]. In prostate cancer patients with SVA and MET combination treatment have less aggression and longer free biochemical recurrence survival than treated only with SVA and MET and suggested may regulate multiple signaling pathways, not only modification of the AMPK, but also androgen receptor (AR) and cyclin-dependent kinase inhibitors (CKIs) [41]. In our study, we found SVA and MET combination inhibited cell growth by decreasing RB and MCM7 expression, and promoted apoptosis by increasing cleaved-caspase 3 and decreasing survival expression.

Angiogenesis is another hallmark of cancer [28]. According to our previously study, use of SVA or MET alone can inhibit tumor angiogenesis and induce vessel normalization [15, 20,43]. In this study, we found synergism of SVA and MET noticeably inhibited angiogenesis of the structure disorder and nonfunctional, and promoted the integrity of vascular structure and functional normalization more than SVA or MET only treatment in tumor of orthotopically transplanted mice. According to our RNA-seq results, ET1 shown a notable downregulation after treatment of synergism of SVA and MET. ET-1 play an important role in mitosis for blood, endothelial cells, fibroblasts, vascular smooth muscle cells and pericytes, and its receptor ETBR also regulated proliferation of blood and endothelial. In melanoma cells, ET-1 promotes tubular network formation through ETBR $[31,44,45]$. Here, we found synergism of SVA and MET could inhibit ET-1 and ETBR expression in vivo and in vitro. Thus, it is an important strategy for anti-tumor by inhibition of ET-1-ETBR axis mediated angiogenesis.

In tumors, hypoxia could induce angiogenesis, which can be regulated by HIF (particularly HIF1a) [7]. Synergism of SVA and MET inhibited HIF1a expression and decreased hypoxia area. ET-1 can stabilize HIF1a by inhibiting PHD2 to control tumor environment [46, 47]. Our results shown an outstanding decline of ET-1 induced ETBR and HIF1a expression, accompanied by an up-regulation of PHD2. However, hypoxia can also upregulate ET-1 expression [48]. Fluvastatin attenuated hypoxia-induced ET-1 transactivation by increasing polyubiquitination of HIF1a and decreasing HIF1a binding to ET-1 promotor [49]. Whether synergism of SVA and MET also could show a more effectively downregulate hypoxiainduced ET-1 expression, consequently inhibit this loop, we will study in our next work.

Bosentan is an antagonist of ETBR, uses in treatment of pulmonary arterial hypertension in clinical, and investigates in Phase II of metastatic melanoma [50,51]. Synergism of SVA and MET displayed a significant influence of anti-tumor effect by regulation of ET-1 induced HIF1a downregulation and PHD2 upregulation than bosentan both in tumor cells and breast cancer PDO. It is suggested that synergism of SVA and MET might be a more effective therapeutic schedule for inhibition of ET-1-Hif1a axis in antitumor treatment. But we just culture 8 breast cancer PDOs, more tumor cases and more tumor types will be need to collect and culture, and combined genomic sequencing and RNA sequencing to screen the other therapeutic target of synergism of SVA and MET, finally use for patients who suit to treat with synergism of SVA and MET in clinical.

\section{Conclusions}


On the hand, our work identifies synergism of SVA and MET as a more effective anti-tumor strategy, compared with ETBR antagonist bosentan. Because of the common problem in development and approval of new drugs, which is a formidably and costly challenge, and often accompanied by a high failure rate. Drug repurposing is an important entry point for drug research and development. Such drugs show a new benefit in medical condition than their original indication [52]. Here, simvastatin and metformin is the first-line clinical medication for reducing hyperglycemia and hyperlipemia. The safety and tolerability have been tested in humans, it will be a huge advantage for reducing time and cost of research and development.

On the other hand, for anti-tumor effect, SVA and MET synergism can alleviate hypoxia and decrease angiogenesis. The improvement of tumor hypoxic microenvironment and tumor angiogenesis can increase the sensitivity of chemotherapy, radiotherapy and tumor immunotherapy, and decrease the characteristics [8,53-55]. Hence, combination of synergism of SVA and MET with the chemotherapy, radiotherapy or immunotherapy can became a potent anti-tumor treatment and improve prognosis options.

\section{Abbreviations}

SVA: Simvastatin; Metformin: MET; PDO: Patient-derived organoid; ET-1: Endothelin 1; HIF1a: Hypoxiainducible factor 1a; PFA: Paraformaldehyde; CFSE: Carboxyfluorescein diacetate succinimidyl ester; PCNA: Proliferating cell nuclear antigen; RB: Retinoblastoma; MCM7: Mini-chromosome maintenance protein 7; CD31: Alternative name of PECAM-1, platelet endothelial adhesion molecule; VEGF: Vascular endothelial growth factor; ETBR: Endothelin receptor B; ETAR: Endothelin receptor A; siRNAs: Small interfering RNAs; PHD2: Prolyl hydroxylase 2; H\&E: Hematoxylin-eosin; TCTP: Translational controlled tumor protein; AR: Androgen receptor; CKIs: cyclin-dependent kinase inhibitors.

\section{Declarations}

\section{Ethics approval and consent to participate}

Breast cancer tissues were obtained from First Affiliated Hospital of Xi 'an Jiaotong University with informed consent and approved by the Ethics Review Committee of the First Affiliated Hospital of Xi 'an Jiaotong University. All animal procedures were performed in accordance with animal care committee of $\mathrm{Xi}$ ' an Jiaotong University. The methods were consistent with the approved guidelines.

\section{Consent for publication}

Not applicable.

\section{Availability of data and materials}


The all authors confirm that the data supporting the findings of this study are available within the article and its supplementary materials.

\section{Competing interests}

The authors declare that they have no conflict of interest.

\section{Funding}

This study was supported by the National Natural Science Foundation of China (No. 81703002 and 81872272), Key Research and Development Program of Shaanxi (No. 2020SF-038 and 2021SF-121) and Clinical Research Award of the First Affiliated Hospital of Xi'an Jiaotong University, China (No. XJTU1AFCRF-2017-007).

\section{Author contributions}

Jie Liu and Peijun Liu conceived and designed the experiments. Jie Liu, Huxia Wang, Miao Zhang, Yazhao Li, Ruiqi Wang, He Chen, Xiaoqian Gao and Shaoran Song performed the experiments and analyzed the data. Bo Wang, Jichang Wang, Yaochun Wang, Juan Li and Peijun Liu contributed reagents and materials. Jie Liu wrote the manuscript. All authors read and approved the final manuscript.

\section{Acknowledgements}

Not applicable.

\section{References}

1. Hanahan D and R A Weinberg. Hallmarks of cancer: the next generation. Cell. 2011;144: 646-74.

2. Herbel C, N Patsoukis, K Bardhan, P Seth, J D Weaver, and V A Boussiotis. Clinical significance of T cell metabolic reprogramming in cancer. Clin Transl Med. 2016;5: 29.

3. Faubert B, A Solmonson, and R J DeBerardinis. Metabolic reprogramming and cancer progression. Science. 2020;368:

4. Vander Heiden M G and R J DeBerardinis. Understanding the Intersections between Metabolism and Cancer Biology. Cell. 2017;168: 657-69.

5. Vander Heiden M G, L C Cantley, and C B Thompson. Understanding the Warburg effect: the metabolic requirements of cell proliferation. Science. 2009;324: 1029-33.

6. Kim J and R J DeBerardinis. Mechanisms and Implications of Metabolic Heterogeneity in Cancer. Cell Metab. 2019;30: 434-46.

7. Pugh C W and P J Ratcliffe. Regulation of angiogenesis by hypoxia: role of the HIF system. Nat Med. 2003;9: 677-84.

8. Keith B, R S Johnson, and M C Simon. HIF1alpha and HIF2alpha: sibling rivalry in hypoxic tumour growth and progression. Nat Rev Cancer. 2011;12: 9-22. 
9. Petrova V, M Annicchiarico-Petruzzelli, G Melino, and I Amelio. The hypoxic tumour microenvironment. Oncogenesis. 2018;7: 10.

10. Hay N. Reprogramming glucose metabolism in cancer: can it be exploited for cancer therapy? Nat Rev Cancer. 2016;16: 635-49.

11. Martinez-Outschoorn U E, M Peiris-Pages, R G Pestell, F Sotgia, and M P Lisanti. Cancer metabolism: a therapeutic perspective. Nat Rev Clin Oncol. 2017;14: 11-31.

12. Demierre M F, P D Higgins, S B Gruber, E Hawk, and S M Lippman. Statins and cancer prevention. Nat Rev Cancer. 2005;5: 930-42.

13. Li J, J Liu, Z Liang, F He, L Yang, P Li, et al. Simvastatin and Atorvastatin inhibit DNA replication licensing factor MCM7 and effectively suppress RB-deficient tumors growth. Cell Death Dis. 2017;8: e2673.

14. Liang Z, W Li, J Liu, J Li, F He, Y Jiang, et al. Simvastatin suppresses the DNA replication licensing factor MCM7 and inhibits the growth of tamoxifen-resistant breast cancer cells. Sci Rep. 2017;7: 41776.

15. Wang J C, X X Li, X Sun, G Y Li, J L Sun, Y P Ye, et al. Activation of AMPK by simvastatin inhibited breast tumor angiogenesis via impeding HIF-1 alpha-induced pro-angiogenic factor. Cancer Sci. 2018;109: 1627-37.

16. Juarez D and D A Fruman. Targeting the Mevalonate Pathway in Cancer. Trends Cancer. 2020;

17. Lee J, A E Yesilkanal, J P Wynne, C Frankenberger, J Liu, J Yan, et al. Effective breast cancer combination therapy targeting BACH1 and mitochondrial metabolism. Nature. 2019;568: 254-8.

18. Cha J H, W H Yang, W Xia, Y Wei, L C Chan, S O Lim, et al. Metformin Promotes Antitumor Immunity via Endoplasmic-Reticulum-Associated Degradation of PD-L1. Mol Cell. 2018;71: 606-20 e7.

19. Liu J, J Li, H Chen, R Wang, $P$ Li, Y Miao, et al. Metformin suppresses proliferation and invasion of drug-resistant breast cancer cells by activation of the Hippo pathway. J Cell Mol Med. 2020;24: 578696.

20. Wang J C, G Y Li, B Wang, S X Han, X Sun, Y N Jiang, et al. Metformin inhibits metastatic breast cancer progression and improves chemosensitivity by inducing vessel normalization via PDGF-B downregulation. J Exp Clin Cancer Res. 2019;38: 235.

21. Sachs N, J de Ligt, O Kopper, E Gogola, G Bounova, F Weeber, et al. A Living Biobank of Breast Cancer Organoids Captures Disease Heterogeneity. Cell. 2018;172: 373-86 e10.

22. Llovet J M, J Zucman-Rossi, E Pikarsky, B Sangro, M Schwartz, M Sherman, et al. Hepatocellular carcinoma. Nat Rev Dis Primers. 2016;2: 16018.

23. Partridge L, M Fuentealba, and B K Kennedy. The quest to slow ageing through drug discovery. Nat Rev Drug Discov. 2020;19: 513-32.

24. Sirtori C R. The pharmacology of statins. Pharmacol Res. 2014;88: 3-11.

25. Adak T, A Samadi, A Z Unal, and S Sabuncuoglu. A reappraisal on metformin. Regul Toxicol Pharmacol. 2018;92: 324-32. 
26. Soriano A F, B Helfrich, D C Chan, L E Heasley, P A Bunn, Jr., and T C Chou. Synergistic effects of new chemopreventive agents and conventional cytotoxic agents against human lung cancer cell lines. Cancer Res. 1999;59: 6178-84.

27. Folkman J. Role of angiogenesis in tumor growth and metastasis. Semin Oncol. 2002;29: 15-8.

28. Kerbel R S. Tumor angiogenesis. N Engl J Med. 2008;358: 2039-49.

29. Ebos J M and R S Kerbel. Antiangiogenic therapy: impact on invasion, disease progression, and metastasis. Nat Rev Clin Oncol. 2011;8: 210-21.

30. Goel S, D G Duda, L Xu, L L Munn, Y Boucher, D Fukumura, et al. Normalization of the vasculature for treatment of cancer and other diseases. Physiol Rev. 2011;91: 1071-121.

31. Rosano L, F Spinella, and A Bagnato. Endothelin 1 in cancer: biological implications and therapeutic opportunities. Nat Rev Cancer. 2013;13: 637-51.

32. Motte S, K McEntee, and R Naeije. Endothelin receptor antagonists. Pharmacol Ther. 2006;110: 386414.

33. van de Wetering M, HE Francies, J M Francis, G Bounova, F lorio, A Pronk, et al. Prospective derivation of a living organoid biobank of colorectal cancer patients. Cell. 2015;161: 933-45.

34. Meng $J$ and $Y$ Zhu. Efficacy of simvastatin plus metformin for polycystic ovary syndrome: A metaanalysis of randomized controlled trials. Eur J Obstet Gynecol Reprod Biol. 2021;257: 19-24.

35. Babcook M A, R M Sramkoski, H Fujioka, F Daneshgari, A Almasan, S Shukla, et al. Combination simvastatin and metformin induces G1-phase cell cycle arrest and Ripk1- and Ripk3-dependent necrosis in C4-2B osseous metastatic castration-resistant prostate cancer cells. Cell Death Dis. 2014;5: e1536.

36. Kim J S, J Turbov, R Rosales, L G Thaete, and G C Rodriguez. Combination simvastatin and metformin synergistically inhibits endometrial cancer cell growth. Gynecol Oncol. 2019;154: 432-40.

37. Seo Y, J Kim, S J Park, J J Park, J H Cheon, W H Kim, et al. Metformin Suppresses Cancer Stem Cells through AMPK Activation and Inhibition of Protein Prenylation of the Mevalonate Pathway in Colorectal Cancer. Cancers (Basel). 2020;12:

38. Antwi S O, Z Li, K Mody, L R Roberts, and T Patel. Independent and Joint Use of Statins and Metformin by Elderly Patients With Diabetes and Overall Survival Following HCC Diagnosis. J Clin Gastroenterol. 2020;54: 468-76.

39. Babcook M A, S Shukla, P Fu, E J Vazquez, M A Puchowicz, J P Molter, et al. Synergistic simvastatin and metformin combination chemotherapy for osseous metastatic castration-resistant prostate cancer. Mol Cancer Ther. 2014;13: 2288-302.

40. Liu S H, J Yu, J F Creeden, J M Sutton, S Markowiak, R Sanchez, et al. Repurposing metformin, simvastatin and digoxin as a combination for targeted therapy for pancreatic ductal adenocarcinoma. Cancer Lett. 2020;491: 97-107.

41. Jimenez-Vacas J M, V Herrero-Aguayo, A J Montero-Hidalgo, P Saez-Martinez, E Gomez-Gomez, A J Leon-Gonzalez, et al. Clinical, Cellular, and Molecular Evidence of the Additive Antitumor Effects of 
Biguanides and Statins in Prostate Cancer. J Clin Endocrinol Metab. 2021;106: e696-e710.

42. Del Campo J A, M Garcia-Valdecasas, A Gil-Gomez, A Rojas, P Gallego, J Ampuero, et al. Simvastatin and metformin inhibit cell growth in hepatitis $C$ virus infected cells via mTOR increasing PTEN and autophagy. PLoS One. 2018;13: e0191805.

43. Wang J C, X Sun, Q Ma, G F Fu, L L Cong, H Zhang, et al. Metformin's antitumour and anti-angiogenic activities are mediated by skewing macrophage polarization. J Cell Mol Med. 2018;

44. Nelson J, A Bagnato, B Battistini, and P Nisen. The endothelin axis: emerging role in cancer. Nat Rev Cancer. 2003;3: 110-6.

45. Spinella F, L Rosano, V Di Castro, S Decandia, M R Nicotra, P G Natali, et al. Endothelin-1 and endothelin-3 promote invasive behavior via hypoxia-inducible factor-1alpha in human melanoma cells. Cancer Res. 2007;67: 1725-34.

46. Spinella F, L Rosano, M Del Duca, V Di Castro, M R Nicotra, P G Natali, et al. Endothelin-1 inhibits prolyl hydroxylase domain 2 to activate hypoxia-inducible factor-1alpha in melanoma cells. PLoS One. 2010;5: e11241.

47. Caprara V, S Scappa, E Garrafa, V Di Castro, L Rosano, A Bagnato, et al. Endothelin-1 regulates hypoxia-inducible factor-1alpha and -2alpha stability through prolyl hydroxylase domain 2 inhibition in human lymphatic endothelial cells. Life Sci. 2014;118: 185-90.

48. Platero-Luengo A, S Gonzalez-Granero, R Duran, B Diaz-Castro, J I Piruat, J M Garcia-Verdugo, et al. An 02-sensitive glomus cell-stem cell synapse induces carotid body growth in chronic hypoxia. Cell. 2014;156: 291-303.

49. Hisada T, M Ayaori, N Ohrui, H Nakashima, K Nakaya, H Uto-Kondo, et al. Statin inhibits hypoxiainduced endothelin-1 via accelerated degradation of HIF-1alpha in vascular smooth muscle cells. Cardiovasc Res. 2012;95: 251-9.

50. Kefford R, J M Beith, G A Van Hazel, M Millward, J M Trotter, D K Wyld, et al. A phase II study of bosentan, a dual endothelin receptor antagonist, as monotherapy in patients with stage IV metastatic melanoma. Invest New Drugs. 2007;25: 247-52.

51. Russo A, G Bronte, S Rizzo, D Fanale, F Di Gaudio, N Gebbia, et al. Anti-endothelin drugs in solid tumors. Expert Opin Emerg Drugs. 2010;15: 27-40.

52. Strittmatter S M. Overcoming Drug Development Bottlenecks With Repurposing: Old drugs learn new tricks. Nat Med. 2014;20: 590-1.

53. Chouaib S, M Z Noman, K Kosmatopoulos, and M A Curran. Hypoxic stress: obstacles and opportunities for innovative immunotherapy of cancer. Oncogene. 2017;36: 439-45.

54. Munn L L and R K Jain. Vascular regulation of antitumor immunity. Science. 2019;365: 544-5.

55. Lytle N K, A G Barber, and T Reya. Stem cell fate in cancer growth, progression and therapy resistance. Nat Rev Cancer. 2018;18: 669-80.

\section{Figures}




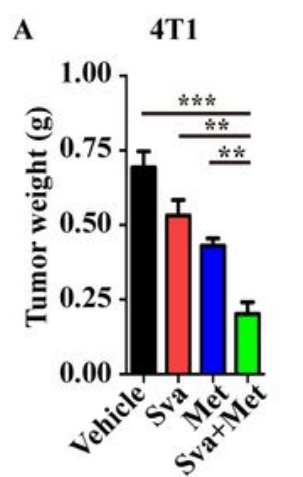

D

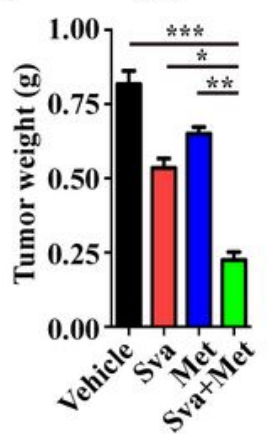

B

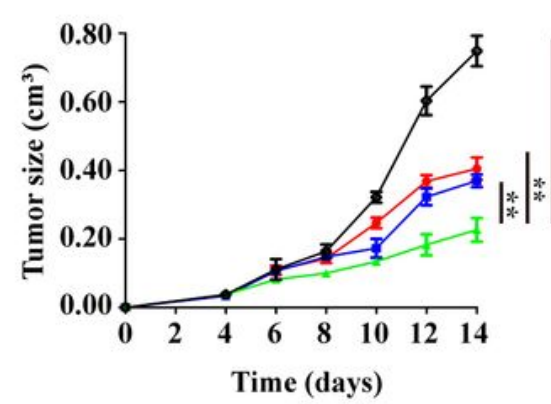

E

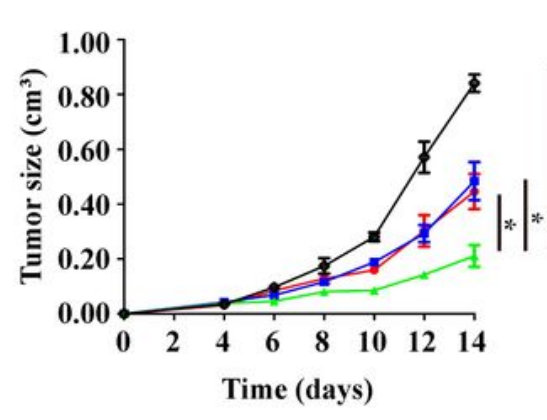

G

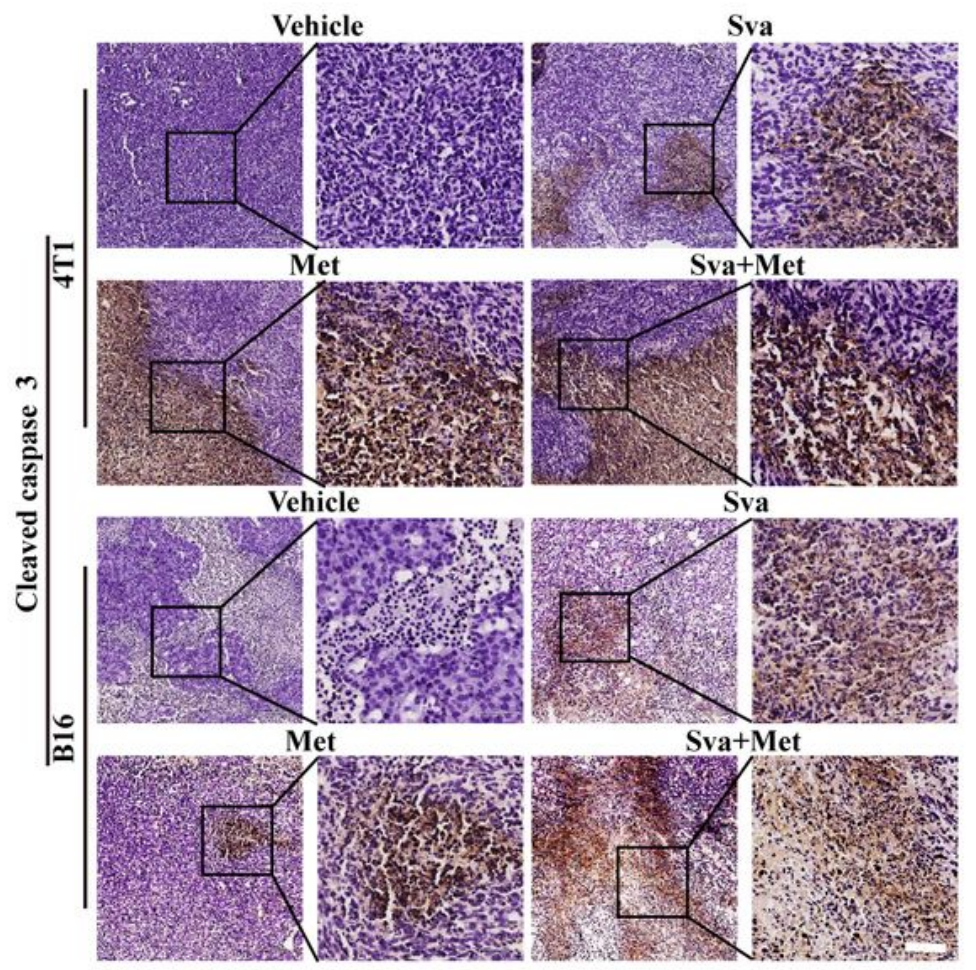

C

$4 \mathrm{~T} 1$
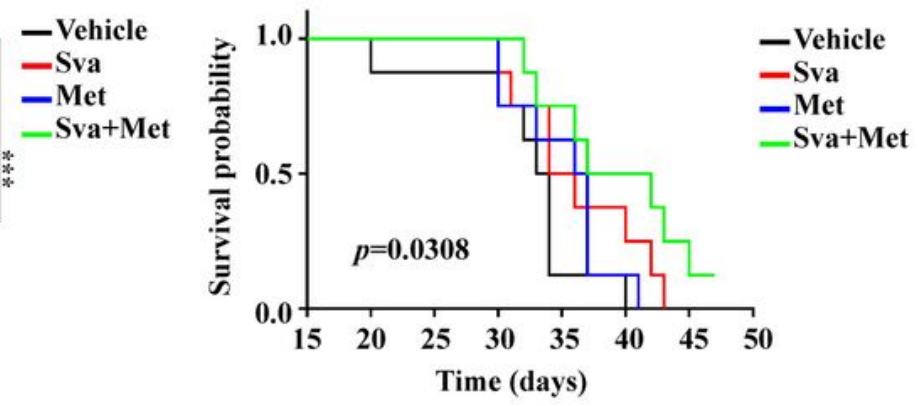

$\mathbf{F}$

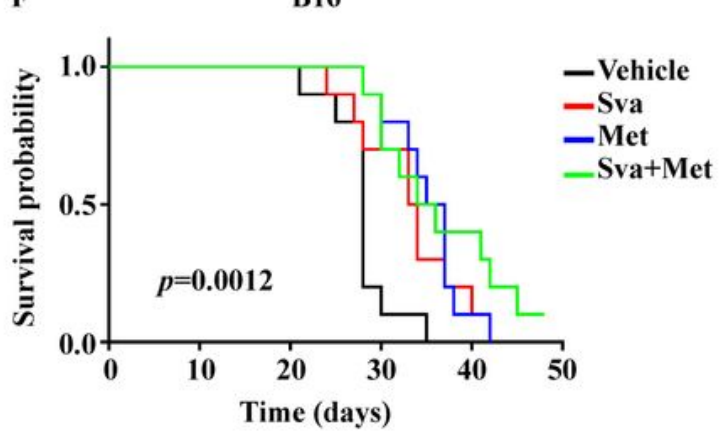

H
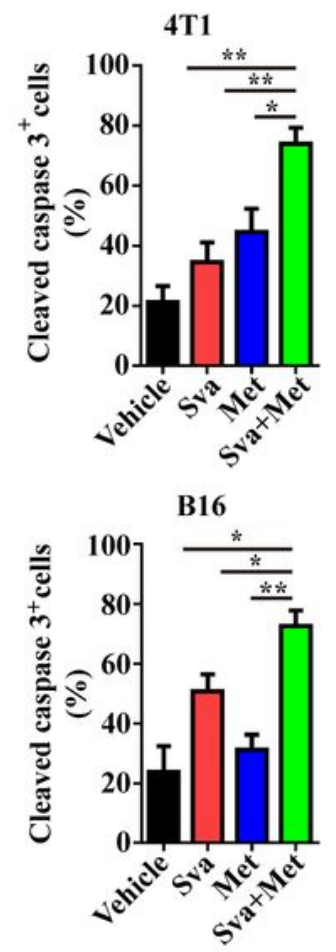

Figure 1

Effects of combination treatment of simvastatin and metformin on tumor growth and mice survival in vivo. Tumor weight (A), tumor growth (B) and mice survival (C) of $4 T 1$ tumor from BALB/c mice, which were untreated or treated with $15 \mathrm{mg} / \mathrm{kg} /$ day SVA, $100 \mathrm{mg} / \mathrm{kg} /$ day MET, and combination of $15 \mathrm{mg} / \mathrm{kg} /$ day SVA and $100 \mathrm{mg} / \mathrm{kg} /$ day MET, $(\mathrm{n}=6)$. Tumor weight $(D)$, tumor growth $(E)$ and mice survival (F) of B16 tumor from C57BL/6J mice, which were untreated or treated with 15mg/kg/day SVA, 100 
$\mathrm{mg} / \mathrm{kg} /$ day MET, and combination of $15 \mathrm{mg} / \mathrm{kg} /$ day SVA and $100 \mathrm{mg} / \mathrm{kg} /$ day MET, $(\mathrm{n}=6) . \mathrm{G}, \mathrm{H}$. Immunochemistry staining of and cleaved caspase 3 of 4 T1 tumor and B16 tumor, Bar: $50 \mu \mathrm{m}$.

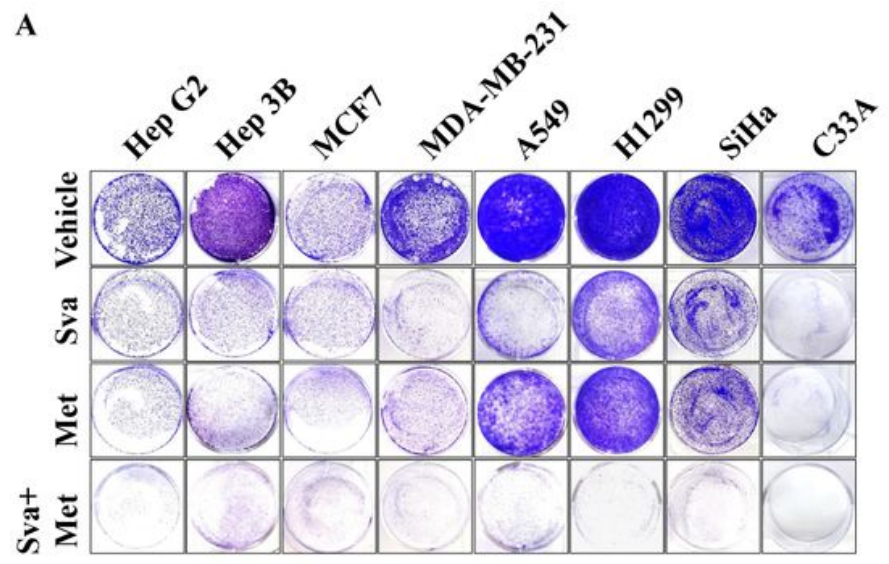

D
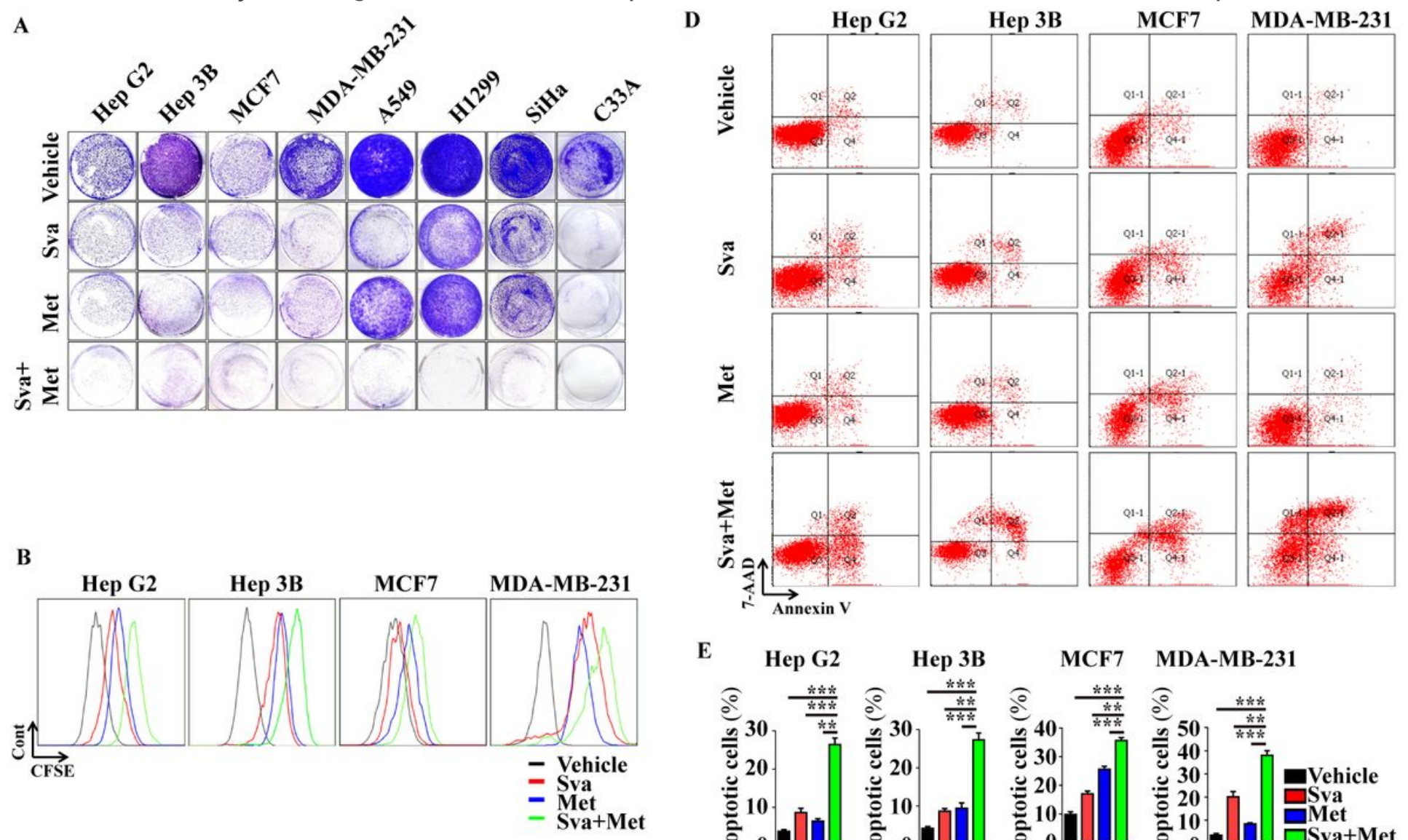

E

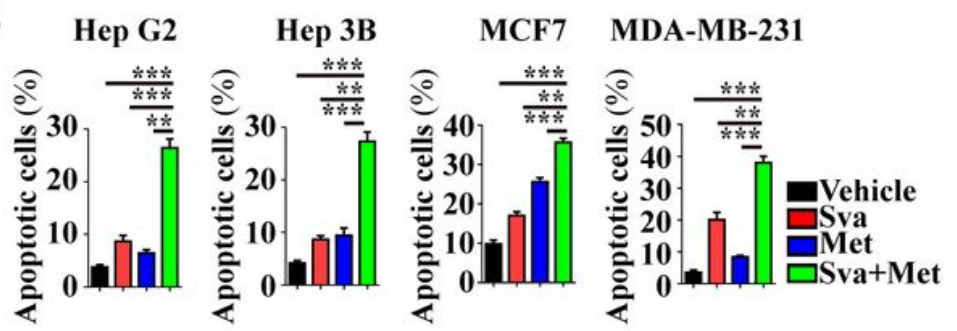

C

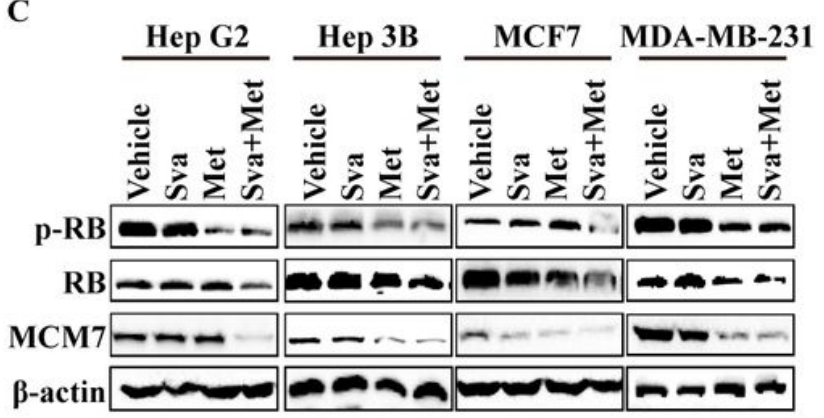

F

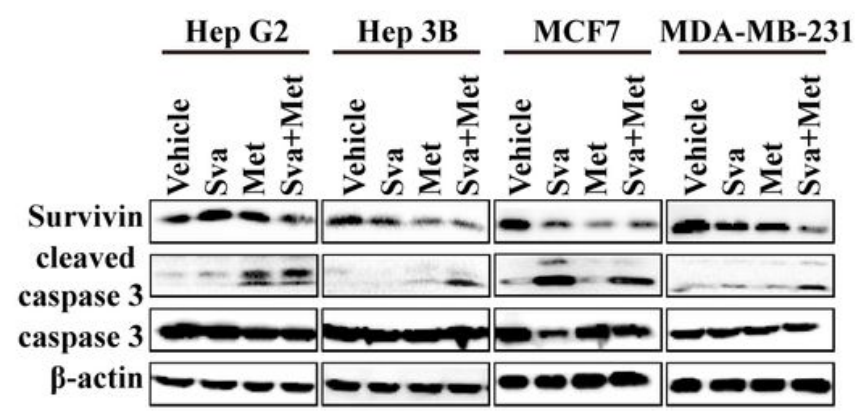

Figure 2

Effects of combination treatment of simvastatin and metformin of different tumor cells. A. Colony formation after 24h of Hep G2, Hep 3B, A549, H1299, SiHa and C33A cells, which were untreated and

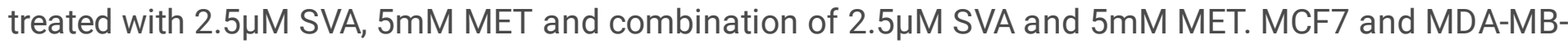
231 cells, which were untreated and treated with $1 \mu \mathrm{M}$ SVA, 10mM MET and combination of $1 \mu \mathrm{M}$ SVA and 10mM MET. B. CFSE assay after 24h of Hep G2 and Hep 3B cells, which were untreated and treated with

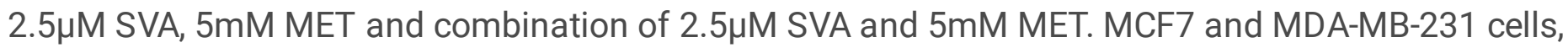
which were untreated and treated with $1 \mu \mathrm{M}$ SVA, $10 \mathrm{mM}$ MET and combination of $1 \mu \mathrm{M}$ SVA and $10 \mathrm{mM}$ MET. C. Western blotting of p-RB, RB and MCM7 of Hep G2, Hep 3B, MCF7 and MDA-MB-231 cells after 
untreated and treated with SVA, MET and combination of and MET. D, E. The percentage of apoptosis ratio of Hep G2, Hep 3B, MCF7 and MDA-MB-231 cells after untreated and treated with SVA, MET and combination of and MET. F. Western blotting of survival, cleaved caspase 3 and caspase 3.

A

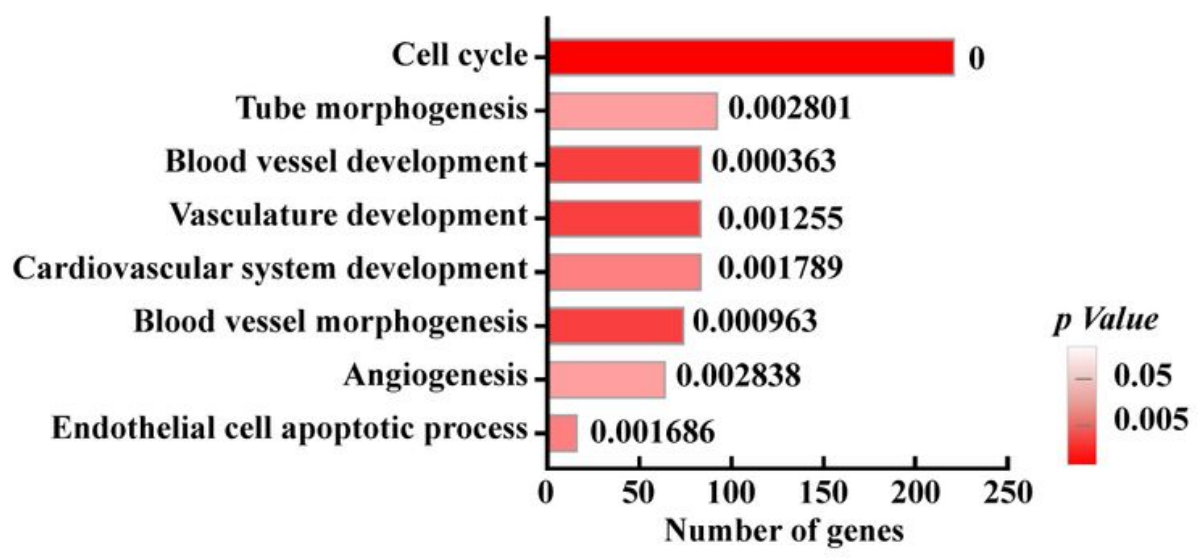

B

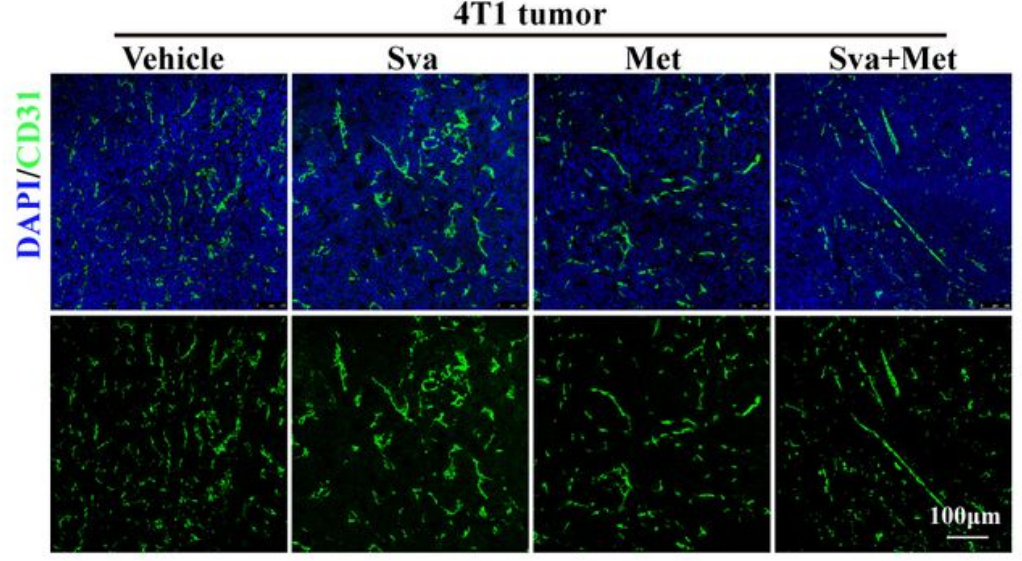

$\mathbf{E}$

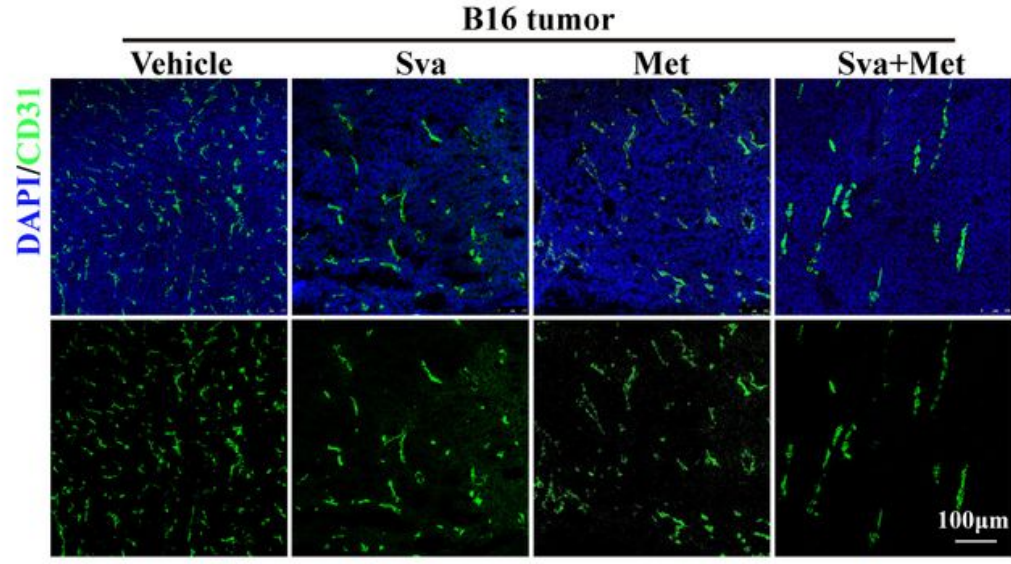

C

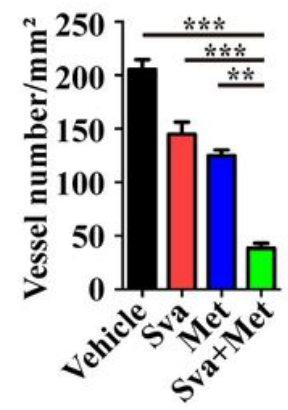

$\mathbf{F}$

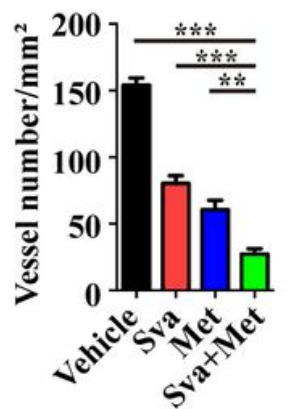

D

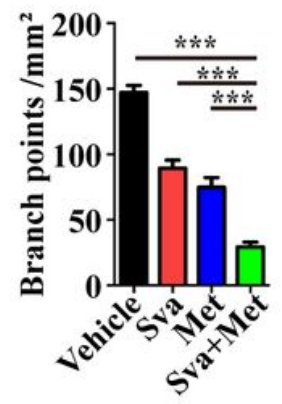

G

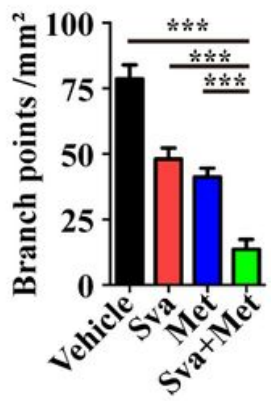

Figure 3

Synergism of simvastatin and metformin regulates vascular morphology and angiogenesis. A. The whole transcriptome of vehicle, SVA, MET and synergism of SVA and MET were identified through RNA sequencing assay. To identify genes were only expressed changes after treatment of synergism of SVA and MET, but no differences in vehicle, SVA or MET by a trend analysis. Top 10 of biological processes were enriched of the genes that express changes only after synergism of SVA and MET treated group by gene ontology (GO) analysis. The 3D-reconstruction of vessels were stained vascular endothelial cell with 
CD31 of 4T1 tumors (B) and B16 tumors (E) after untreated, SVA, MER and synergism of SVA and MET. Bar $=100 \mu \mathrm{m}$. The vessel number of untreated, SVA, MER and synergism of SVA and MET of 4T1 tumors (C) and B16 tumors (F). The statistical analysis of vessel with branch of 4T1 tumors (D) and B16 tumors (G).

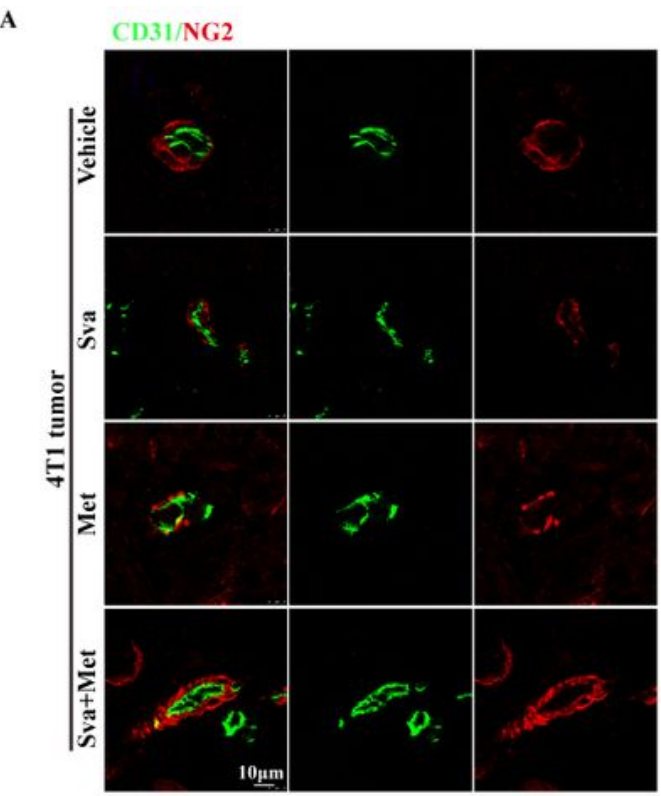

C

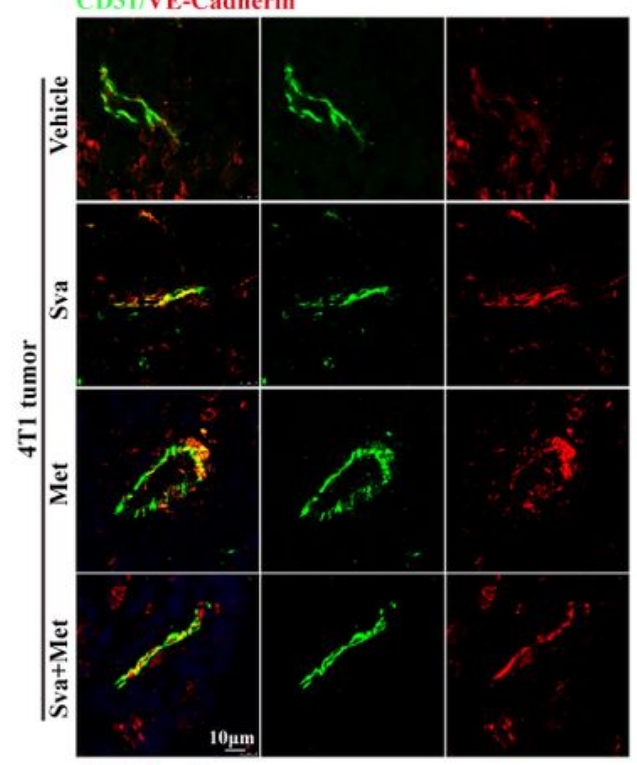

E

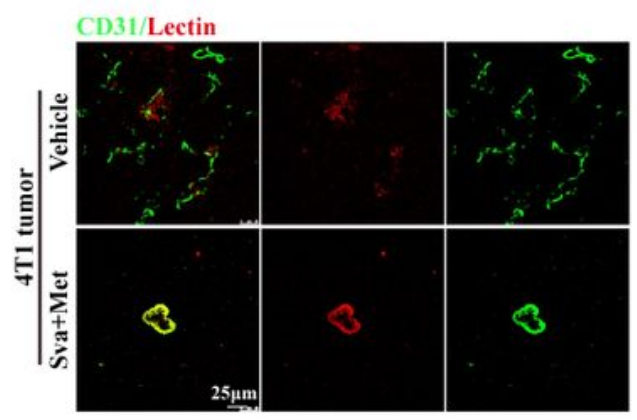

B

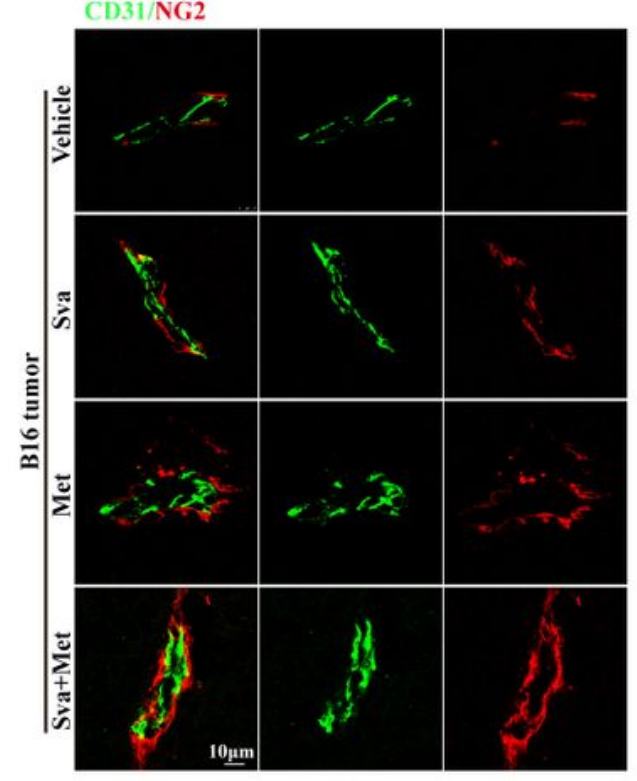

D

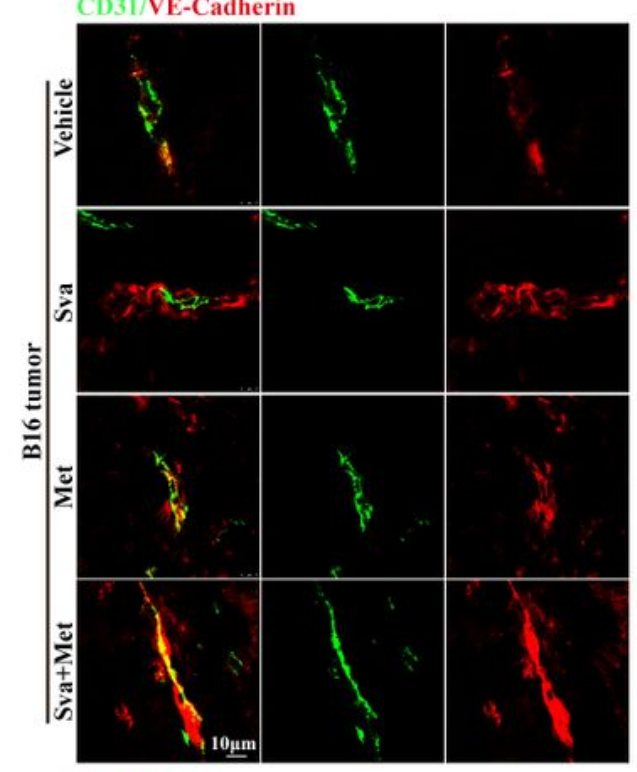

F

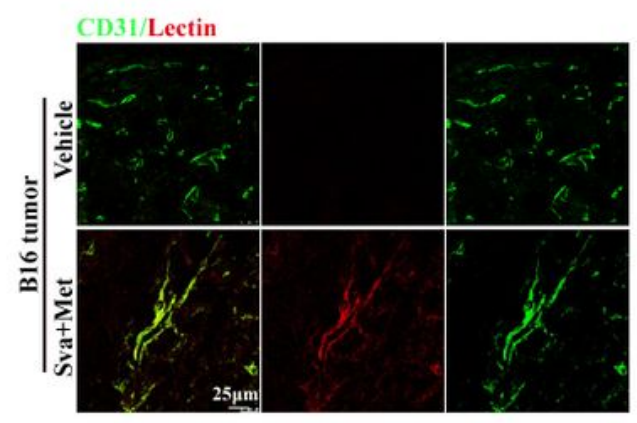

Figure 4 
Vessel maturity and functional status after treatment of synergism of SVA and MET. The immunofluorescence of CD31 (green) and NG2 (red) of frozen section of 4T1 tumors (A) and B16 tumors (B) after untreated, SVA, MER and synergism of SVA and MET. Bar=10 $\mu \mathrm{m}$. The immunofluorescence of CD31 (green) and VE-cadherin (red) of frozen section of 4T1 tumors (C) and B16 tumors (D) after untreated, SVA, MER and synergism of SVA and MET. Bar=10 $\mu \mathrm{m}$. The lectin-perfused CD31+ vessels in frozen section of 4T1 tumors (E) and B16 tumors (F) after untreated and synergism of SVA and MET. Bar $=25 \mu \mathrm{m}$.
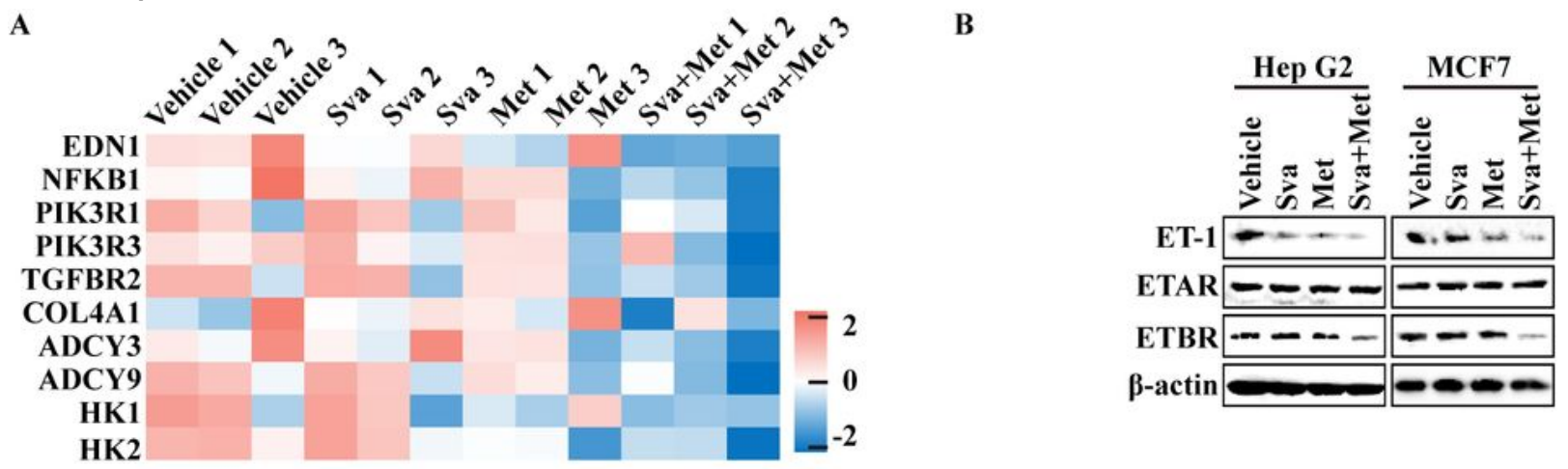

C CD31/ET-1/DAPI

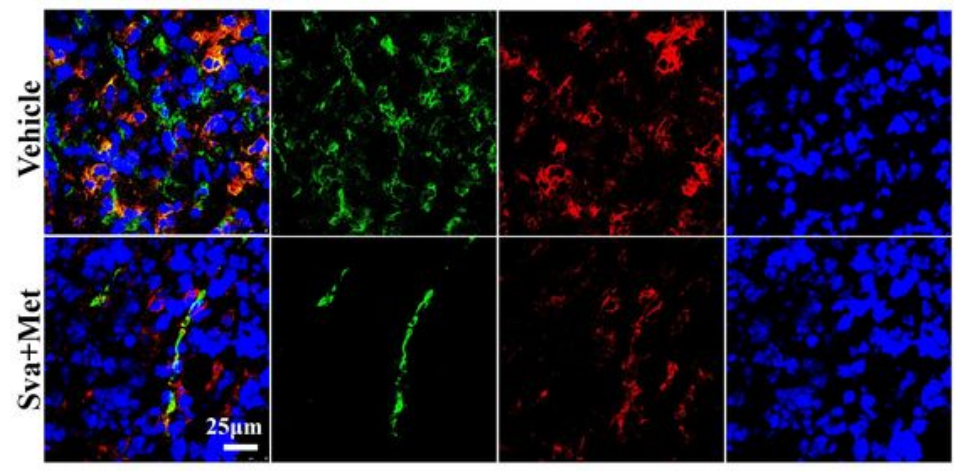

$\mathbf{E}$

4T1 tumor

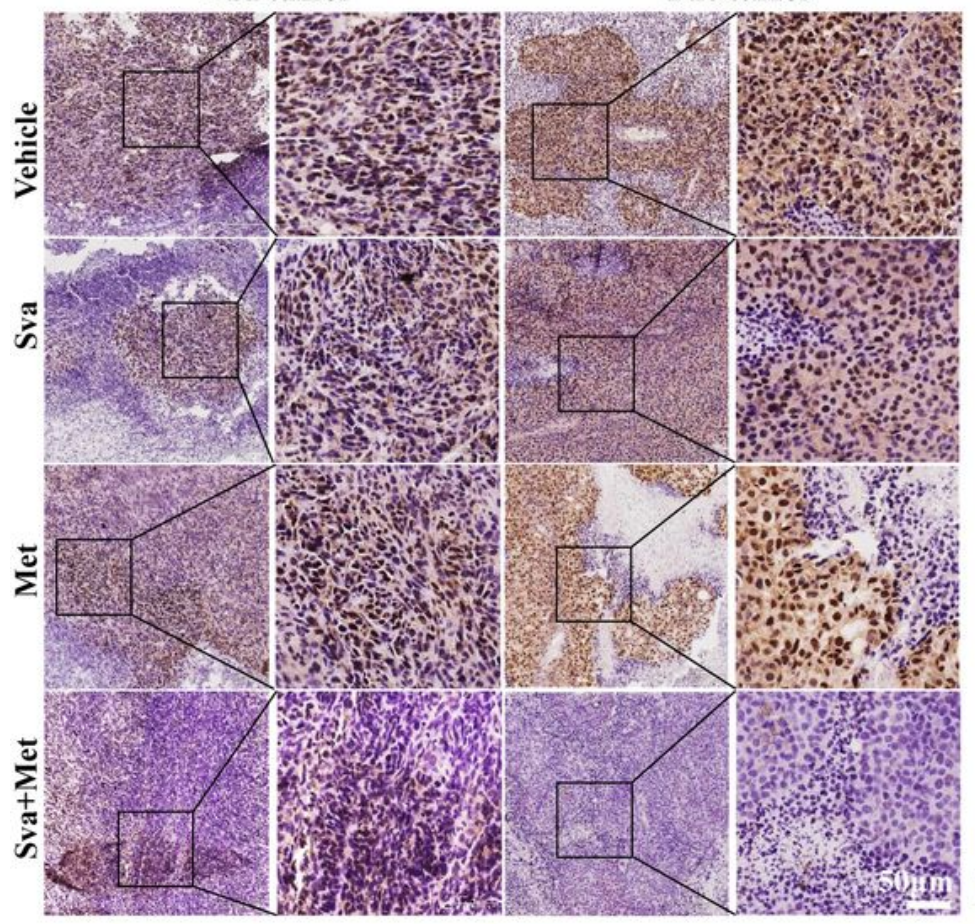

D CD31/ETBR/DAPI

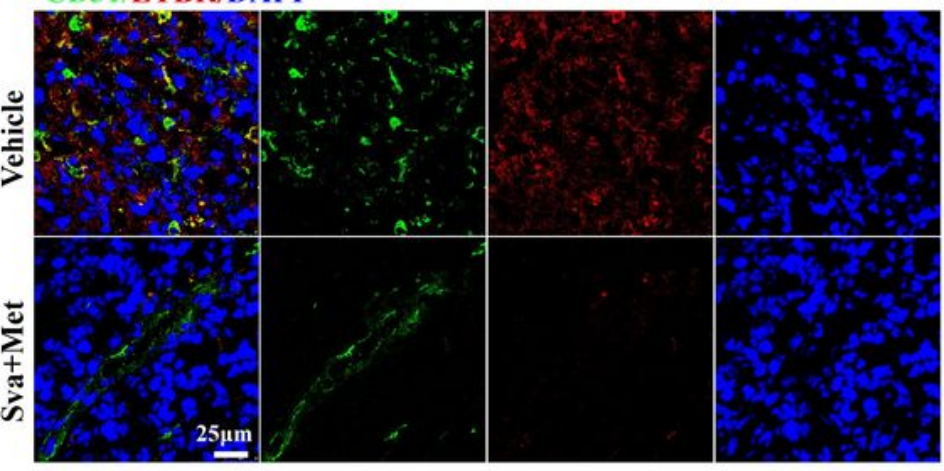

F

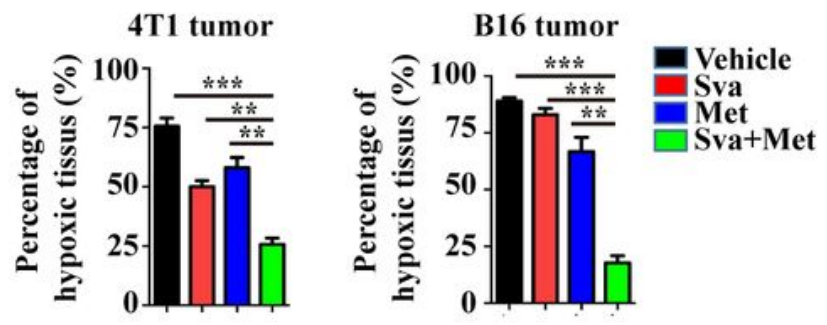

G

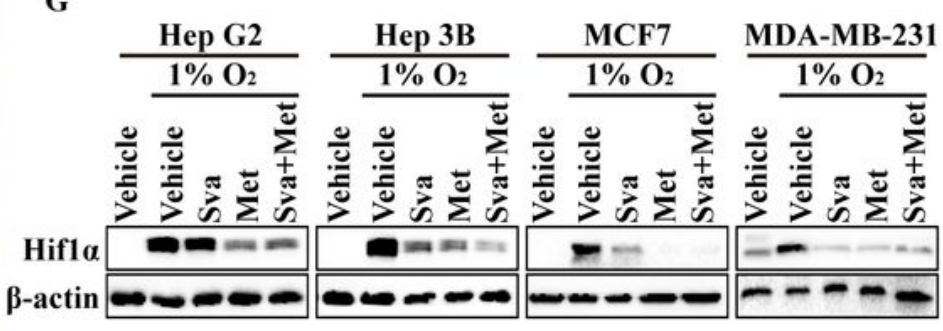




\section{Figure 5}

The effects of hypoxia and ET-1 expression by synergism of SVA and MET. A. The heat map of synergism of SVA and MET regulated hypoxia related genes. B. Western blotting of ET-1, ETAR and ETBR of Hep G2 and MCF7 cells after untreated and treated with SVA, MET and synergism of SVA and MET. The immunofluorescence of CD31 (green) and ETBR (red) of frozen section of 4T1 tumors (C) and B16

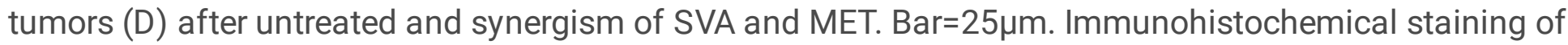
Hif1a (E) and quantification of hypoxic area (F) of 4T1 tumors and tumors. Bar=50 $\mu \mathrm{m}$. G. Western blotting of Hif1a of Hep G2, Hep3B, MCF7 and MDA-MB-231 cells after untreated and treated with SVA, MET and synergism of SVA and MET.

A
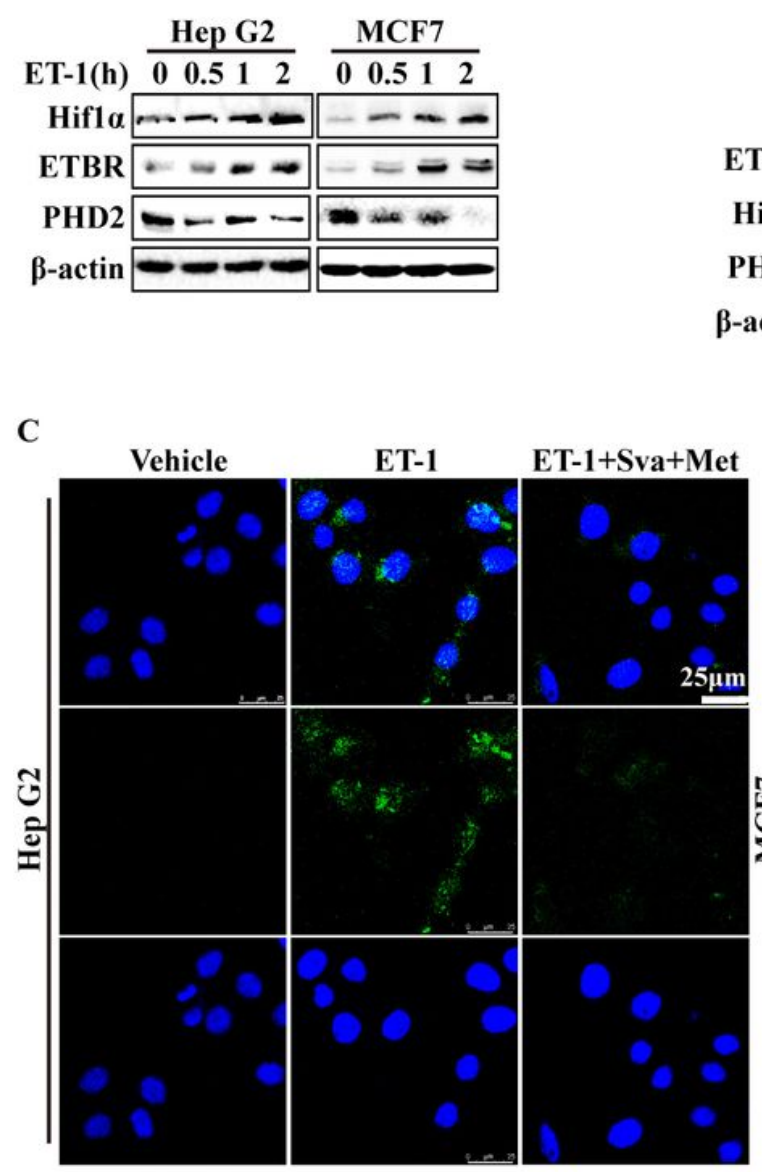

B

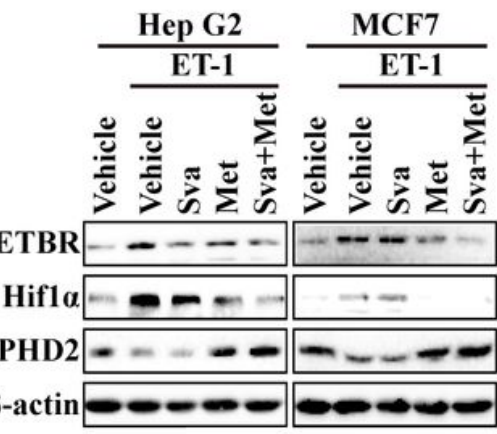

D

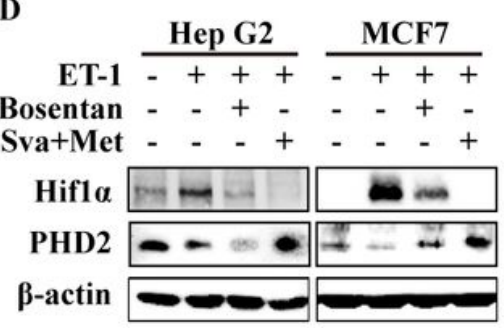

E

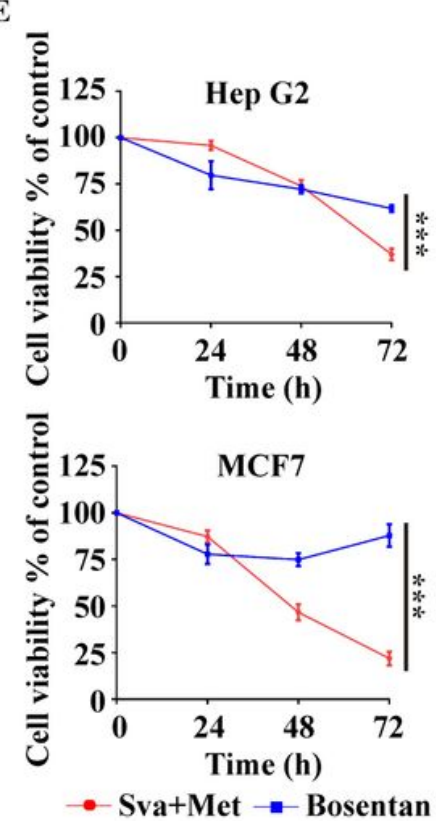

- Sva+Met - Bosentan

Hifl $\alpha /$ DAPI
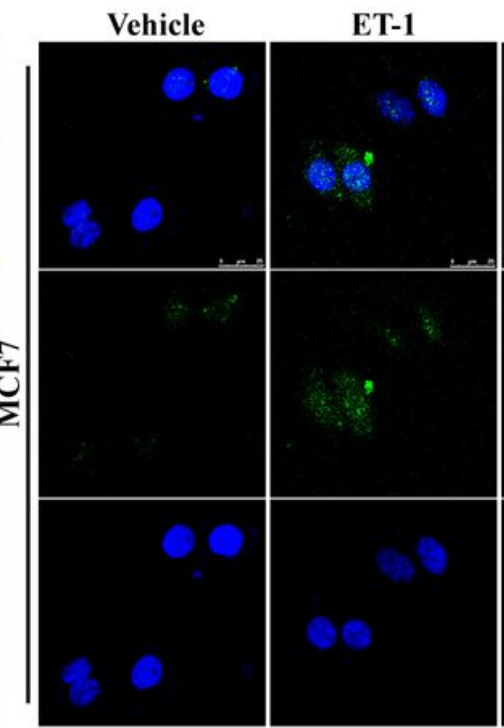

ET-1+Sva+Met

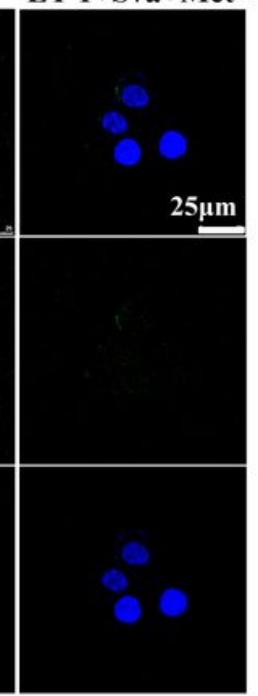

\section{Figure 6}

Synergism of SVA and MET inhibits ET-1-ETBR- Hif1a signaling axis. A. The expression of Hif1a, ETAR, ETBR and PHD2 of Hep G2 and MCF7 cells after treated with 100nM endothelin 1 for $0,0.5,1$ and $2 \mathrm{~h}$ by western blotting. B. Analysis of Hif1a, ETBR and PHD2 expression of Hep G2 and MCF7 cells after pretreated with $100 \mathrm{nM}$ endothelin 1 for $1 \mathrm{~h}$, then treated with SVA, MET and synergism of SVA and MET for 24h by western blotting. C. Immunofluorescence of Hif $1 \mathrm{a}$ after100nM endothelin 1 pre-treated and synergism of SVA and MET. Bar=25 $\mu$ m. D. Analysis of Hif1a, ETBR and PHD2 expression of Hep G2 and 
MCF7 cells after pre-treated with 100nM endothelin 1 for $1 \mathrm{~h}$, then treated with 100nM bosentan and synergism of SVA and MET for $24 \mathrm{~h}$ by western blotting. E. The cell viability curve of Hep G2 and MCF7 cells after treatment with synergism of SVA and MET or bosentan for $24,48,72 \mathrm{~h}$.

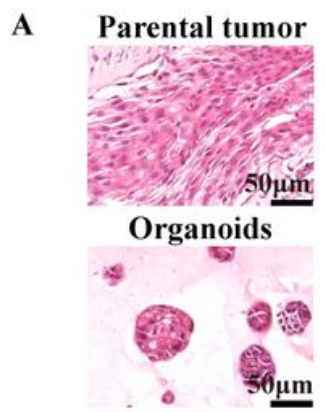

C

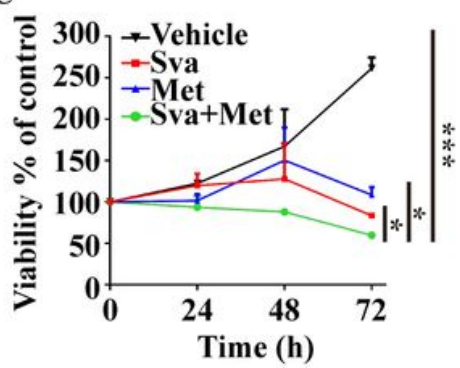

G

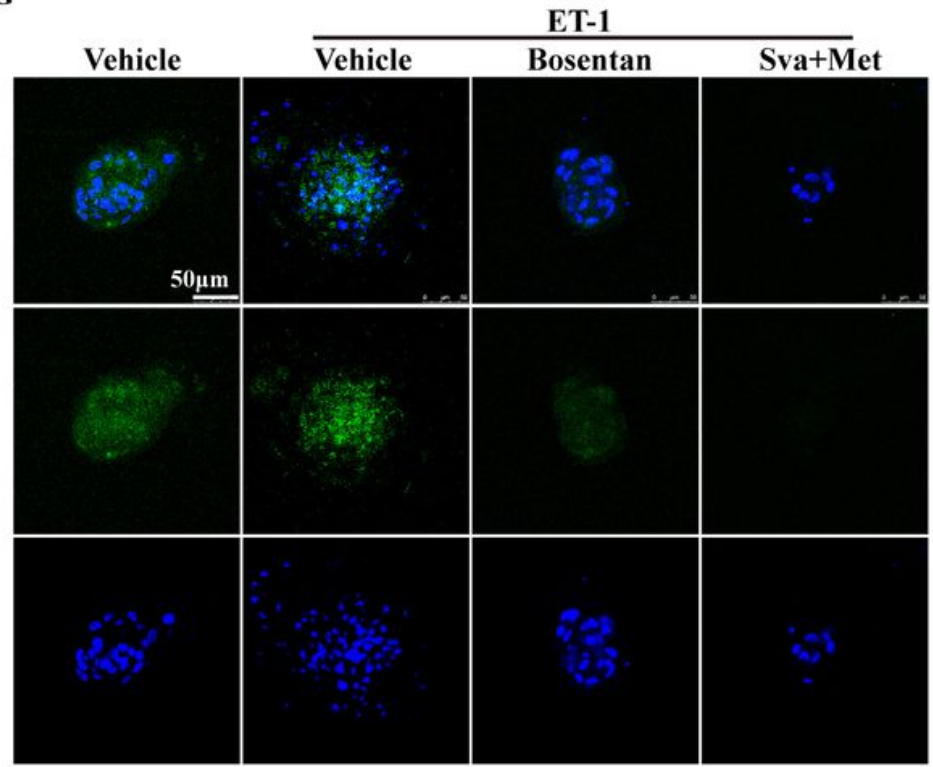

E

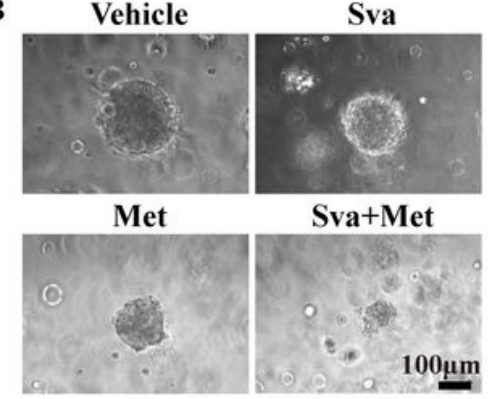

D

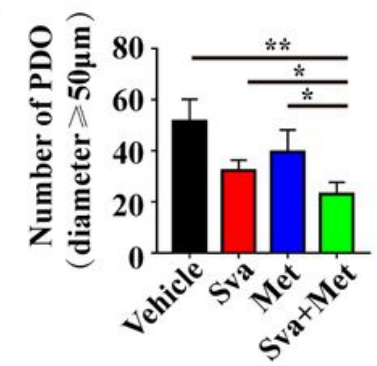

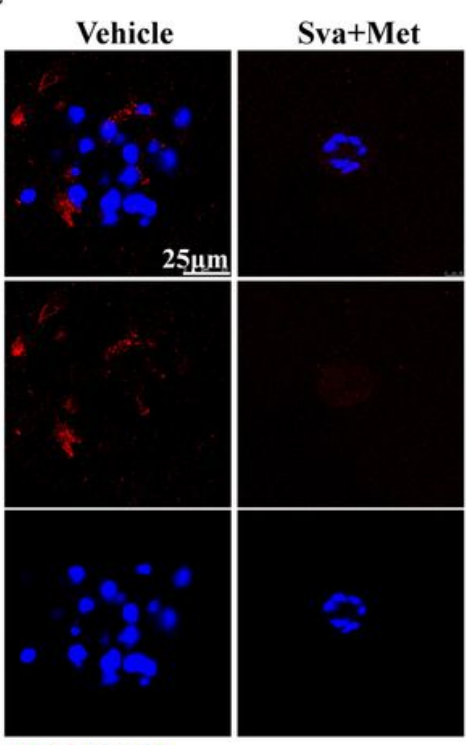

ET-1/DAPI

$\mathbf{H}$
F

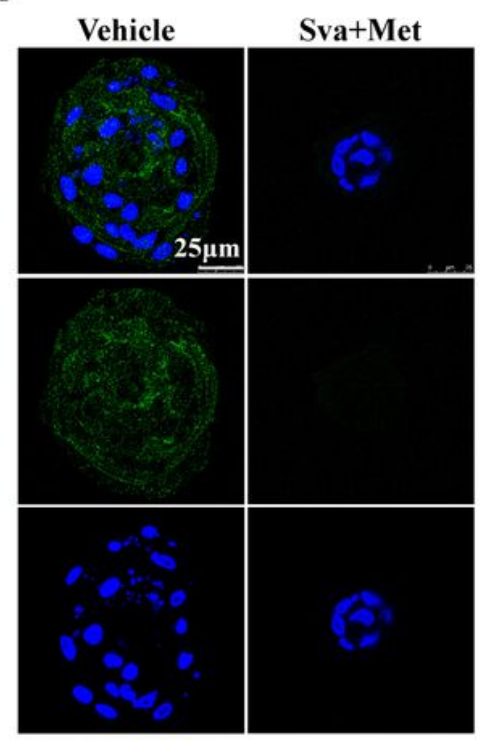

ETBR/DAPI

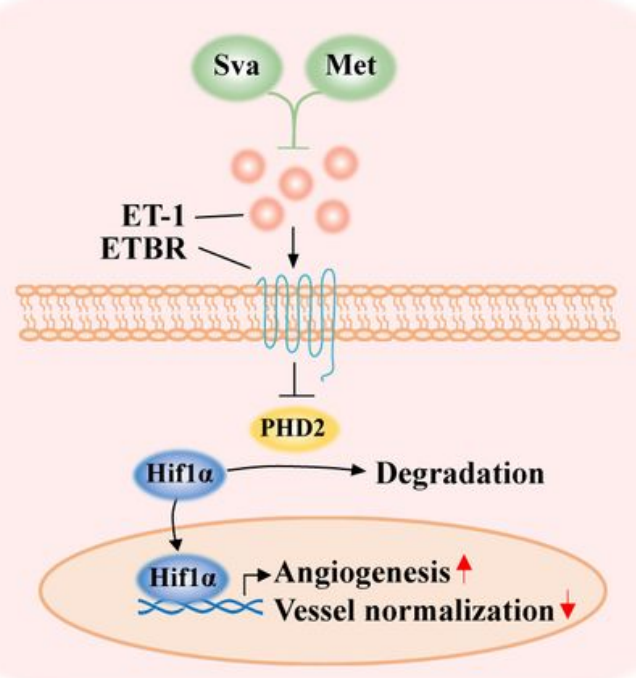

Hifl $\alpha$ DAPI

\section{Figure 7}

Synergism of SVA and MET inhibits growth of breast cancer PDO and ETBR and Hif1a expression. A. H\&E staining of primary breast cancer tumor and PDO derived of it. Bar $=50 \mu \mathrm{m}$. B. The images of PDO after untreated and treated with SVA, MET and synergism of SVA and MET for 72h. The growth (C) and quantification of volume (D) of breast cancer PDOs (diameter $\geq 50 \mu \mathrm{m}$ ) after untreated and treated with SVA, MET and synergism of SVA and MET for 72h. Immunofluorescence of ET-1 (E, red) and ETBR (F, green) of PDO after untreated and treated with synergism of SVA and MET for $72 \mathrm{~h}$. Bar=25 $\mu \mathrm{m}$. G. Immunofluorescence of Hif1a (green) after pre-treated with 100nM endothelin $1 \mathrm{for} 1 \mathrm{~h}$, then untreated 


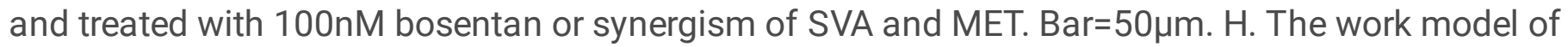
synergism of SVA and MET regulated the ET-1-ETBR- Hif1a signaling axis to inhibition of angiogenesis and promotion vessel normalization.

\section{Supplementary Files}

This is a list of supplementary files associated with this preprint. Click to download.

- supplementtarymaterial.docx 